\title{
High-field vortices in Josephson junctions with alternating critical current density
}

\author{
M. Moshe and R. G. Mint:* \\ School of Physics and Astronomy, Raymond and Beverly Sackler \\ Faculty of Exact Sciences, Tel Aviv University, Tel Aviv 69978, Israel
}

(Dated: November 30, 2018)

\begin{abstract}
We study long Josephson junctions with the critical current density alternating along the junction. New equilibrium states, which we call the field synchronized or FS states, are shown to exist if the applied field is from narrow intervals centered around equidistant series of resonant fields, $H_{m}$. The values of $H_{m}$ are much higher than the flux penetration field, $H_{s}$. The flux per period of the alternating critical current density, $\phi_{i}$, is fixed for each of the FS states. In the $m$-th FS state the value of $\phi_{i}$ is equal to an integer amount of flux quanta, $\phi_{i}=m \phi_{0}$. Two types of single Josephson vortices carrying fluxes $\phi_{0}$ or/and $\phi_{0} / 2$ can exist in the FS states. Specific stepwise resonances in the current-voltage characteristics are caused by periodic motion of these vortices between the edges of the junction.
\end{abstract}

PACS numbers: 74.50.+r, 74.78.Bz, 74.81.Fa

Keywords: $\pi$-shifted Josephson junction, Josephson vortex

Josephson tunnel structures arranged in a sequence of interchanging 0 - and $\pi$-shifted fragments (see Fig. (1) are a subject of growing interest $1,2,3,4,4,5,6,7$, 8, 9, 10, 11, 12, 13, 14, 15]. Currently, the properties of these complex systems are thoroughly treated for superconductor-ferromagnet-superconductor (SFS) heterostructures [1, 2, 3, 4, [5] and asymmetric grain boundaries in thin films of high- $\mathrm{T}_{c}$ superconductor $\mathrm{YBCO}$ $[6,7,8,19,10,11,12,13,14,15]$.

It has been predicted that in the SFS Josephson junctions the $\pi$-shift in the phase difference $\varphi$ between the superconducting banks is caused by the ferromagnet interlayer [1, 2]. This prediction was confirmed in recent experiments with SFS $\pi$-shifted junctions and SFS heterostructures of interchanging 0 - and $\pi$-shifted fragments [3, 4, 5]. The asymmetric grain boundaries in YBCO thin films are arranged in series of facets with variety of orientations and lengths $l \sim 10-100 \mathrm{~nm}[10]$. This spatial structure in conjunction with the $d$-wave symmetry of the order parameter results in grain boundary junctions with interchanging 0 - and $\pi$-shifted fragments [8, 9].

The critical current density, $j_{c}(x)$, changes sign at each

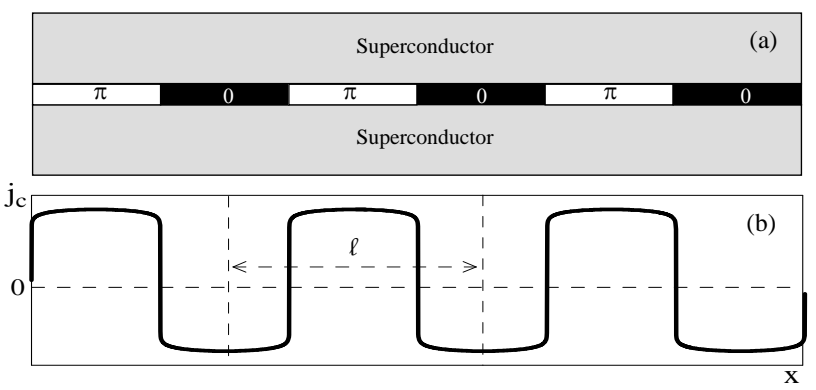

FIG. 1: Schematic diagrams of: (a) tunnel junction arranged in series of interchanging 0 - and $\pi$-shifted fragments; (b) spatial distribution of alternating critical current density. contact between 0 - and $\pi$-shifted fragments ( $x$ axis is along the junction). This results in a variety of dramatic changes in Josephson properties. in particular, the field dependence of the maximum supercurrent across the junction, $I_{\mathrm{m}}$, is strongly affected by the alternating $j_{c}(x)$ 6, 7, 12, 15]. First, the value $I_{m}\left(H_{a}\right)$ is significantly suppressed at low fields, $\left|H_{a}\right| \ll H_{1}=\phi_{0} / 2 \lambda l$, where $\lambda$ is the London penetration depth and $l$ is the period of $j_{c}(x)$. Second, unlike the standard Fraunhofer pattern with a major peak at $H_{a}=0$, two major side-peaks are observed at high fields, $H_{a}= \pm H_{1}$, where $H_{1} \gg H_{s}$ and $H_{s}$ is the field of first flux penetration [7].

In this letter we find a series of new equilibrium field synchronized (FS) states existing if the applied field is from narrow intervals $\Delta H_{a} \sim H_{s}$ centered at the resonant fields $H_{m}= \pm m H_{1}$, where $m \neq 0$ is an integer. It is shown that in the $m$-th FS state the flux per period of the alternating critical current density, $\phi_{i}$, is fixed and is equal to an integer number of flux quanta, $\phi_{i}=m \phi_{0}$. We demonstrated that two types of high-field $\left(H_{a} \gg H_{s}\right)$ Josephson vortices with fluxes $\phi_{0}$ or/and $\phi_{0} / 2$ can exist in the FS states. We find that periodic motion of these single high-field vortices cause stepwise resonances in the IV curves similar to the zero-field resonances in Josephson junctions of conventional superconductors [16].

We begin with a qualitative treatment of the FS states using one harmonics model for the tunneling current density $j=j_{c}(x) \sin \varphi$, where $j_{c}(x)=j_{1} \sin (2 \pi x / l), L=N l$, $L$ is the length of the junction, and $N \gg 1$ is an integer. Assume that the junction is in one of the FS states and flux $\phi_{i}$ is fixed. Since we have many vortices in the junction $(N \cdot m \gg 1)$, the field is almost uniform and the phase $\varphi(x)$ takes the form

$$
\varphi(x)=2 \pi \frac{\phi_{i}}{\phi_{0}} \frac{x}{l}+\psi(x),
$$

where the phase $\psi(x)$ is a smooth function with the typ- 
ical length scale $\gg l$ and $|\psi(x)| \sim 1$. Then we have:

$$
j(x)=j_{1} \sin \left(2 \pi \frac{x}{l}\right) \sin \left(2 \pi \frac{\phi_{i}}{\phi_{0}} \frac{x}{l}+\psi\right) .
$$

In general, this current density alternates rapidly with a typical length scale $\leq l$. In this case the coarse-grained approach is the right tool to describe the smooth phase $\psi(x)$ [13]. If we average $j(x)$ over a distance $\mathcal{L} \gg l$, then the coarse-grained tunneling current density, $j_{\psi}$, is zero. This is indeed true in all cases but one. If the junction is in the FS state with $\phi_{i}= \pm \phi_{0}$, then coarse-graining of Eq. (2) leads to a nonzero result

$$
j_{\psi}=0.5 j_{1} \sin (\psi \pm \pi / 2) .
$$

It is worth mentioning that $j_{\psi} \sim j_{1}$ and the final form of the dependence of $j_{\psi}$ on the smooth phase $\psi$ coincides with a $\pi / 2$-shifted current-phase relation of a Josephson junction of conventional superconductors.

Similar calculation of the coarse-grained current density $j_{\psi}$ in the low-field region $\left(H_{a} \ll H_{s}\right)$ leads to [13]

$$
j_{\psi}=-j_{1}\left(\frac{l}{4 \pi \Lambda_{1}}\right)^{2} \sin 2 \psi \ll j_{1}
$$

where the Josephson length is given by

$$
\Lambda_{1}=\sqrt{c \phi_{0} / 16 \pi^{2} \lambda j_{1}} \gg l .
$$

Comparing Eqs. (2), (3), and (4) we find that if the phase factor, $\sin \varphi$, modulations caused by the field and the critical current density modulations caused by the structure of the junction are synchronized $\left(\phi_{i}=m \phi_{0}\right)$, then the critical current density $j_{c}(x)$ is significantly enhanced (see Fig. (2). The widths of the intervals $\Delta H_{a} \sim$ $H_{s} \ll H_{1}$ of existence of the FS states are defined by minimization of the free energy of the junction.

Consider now a Josephson junction with $\lambda \ll l \ll \lambda_{J}$, where $\lambda_{J}$ is the local Josephson penetration depth

$$
\lambda_{J}=\sqrt{c \phi_{0} / 16 \pi^{2} \lambda\left\langle\left|j_{c}\right|\right\rangle}
$$

and the averaging over the junction length is defined as

$$
\langle f\rangle=\int_{0}^{L} d x f(x) / L .
$$

In this case the static spatial distribution of the phase $\varphi(x)$ is given by

$$
\lambda_{J}^{2} \varphi^{\prime \prime}-i_{c}(x) \sin \varphi=0,
$$

where $i_{c}(x)=j_{c}(x) /\left\langle\left|j_{c}\right|\right\rangle$ is the dimensionless critical current density. Next, we expend $i_{c}(x)$ in Fourier series and obtain instead of Eq. (8):

$$
\lambda_{J}^{2} \varphi^{\prime \prime}-\sum_{n=-\infty}^{\infty} i_{n} e^{i 2 \pi n x / l} \sin \varphi=0
$$

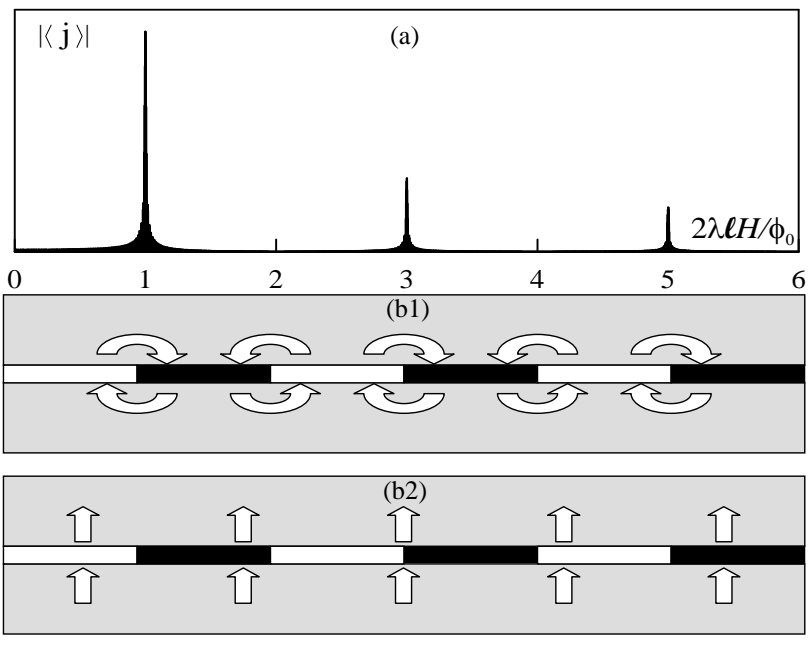

FIG. 2: (a) Coarse-grained tunneling current density dependence on the applied field for the case of a stepwise critical current density, $N=40$, and $\varphi \propto x$. Schematic diagrams of spatial distributions of tunneling current density for junctions that are: (b1) not in the FS states (small current loops tend to cancel each other); (b2) in the FS states (field synchronized local currents flow in the same direction).

where $i_{n}$ are the Fourier transforms of the function $i_{c}(x)$. Since $l \ll \lambda_{J}$, the phase $\varphi$ can be written as $[13$, 17]

$$
\varphi=2 \pi \phi_{i} x / \phi_{0} l+\psi(x)+\xi(x),
$$

where $\psi(x)$ is a smooth function with the typical length scale $\sim \lambda_{J} \gg l$ and $|\psi(x)| \sim 1, \xi(x)$ is a rapidly alternating function with the length scale $\sim l$. In addition, we assume that $\langle\xi(x)\rangle=0$, and $\langle|\xi(x)|\rangle \ll 1$. Following [13, 17] we average Eq. (9) over the junction length and obtain the equation describing the smooth (coarsegrained) phase $\psi(x)$ in the $m$-th FS state

$$
\lambda_{J}^{2} \psi^{\prime \prime}=\left|i_{m}\right| \sin \left(\psi-\theta_{m}\right)-\gamma_{m} \sin 2\left(\psi-\alpha_{m}\right),
$$

where $\theta_{m}=\arg \left(i_{m}\right), \alpha_{m}$ and $\gamma_{m}$ are defined by

$$
\gamma_{m} e^{-i 2 \alpha_{m}}=\left(\frac{l}{2 \pi \lambda_{J}}\right)^{2} \sum_{n=1}^{\infty} \frac{i_{m+n} i_{m-n}}{n^{2}} .
$$

In general, complexity of Eq. (11) can be significantly reduced. Indeed, one can estimate $\gamma_{m} \sim\left(l / 2 \pi \lambda_{J}\right)^{2} \ll 1$, which means that $\gamma_{m} \ll i_{n}$. As a result the second term in the RHS of Eq. (11) can be neglected and Eq. (11) yields the sine-Gordon equation

$$
\Lambda_{m}^{2} \psi^{\prime \prime}=\sin \left(\psi-\theta_{m}\right),
$$

where $\Lambda_{m}=\lambda_{J} / \sqrt{\left|i_{m}\right|}$. The boundary conditions to Eq. (13) are given by the field at $x=0$ and $x=L$ :

$$
\left.\psi^{\prime}\right|_{0, \mathrm{~L}}=\frac{4 \pi \lambda}{\phi_{0}}\left(\left.H\right|_{0, \mathrm{~L}}-m H_{1}\right) .
$$


It is worth noting that, in particular, Eq. (13) describes Josephson-type vortices with size $\sim \Lambda_{m} \gg l$ and flux $\phi_{0}$.

It follows from Eqs. (11) and (13) that equations describing the coarse-grained phase $\psi(x)$ are the same as for Josephson junctions of conventional superconductors in the Meissner state.

Specific symmetry of $j_{c}(x)$ might lead to a Fourier series with some of the transforms being zero. In this case only the second term at the RHS of Eq. (11) is non zero. In particular, if $j_{c}(x)$ is a stepwise function (see Fig. 1), then we have $i_{2 \mathrm{k}}=0$ and $i_{2 \mathrm{k}+1}=-2 i / \pi(2 k+1)$, where $k$ is an integer. In the FS states with the field $H_{a}$ located in the intervals $\Delta H_{a}$ centered at "odd" resonant fields $H_{\mathrm{ok}}=(2 k+1) H_{1}$, Eq. (11) takes the form

$$
\Lambda_{\mathrm{ok}}^{2} \psi^{\prime \prime}=\sin \psi, \quad \Lambda_{\mathrm{ok}}=\sqrt{\pi(k+1 / 2)} \lambda_{J} .
$$

Similarly, for "even" resonant fields $H_{\mathrm{ek}}=2 k H_{1}$, Eq. (11) yields

$$
\Lambda_{\mathrm{ek}}^{2} \psi^{\prime \prime}=\sin 2 \psi, \quad \Lambda_{\mathrm{ek}}=2 \pi k \beta_{k} \frac{\lambda_{J}^{2}}{l},
$$

where $\beta_{k}$ is a constant $\left(\beta_{1}=0.9, \beta_{2}=1.1, \beta_{3}=0.9\right.$, and $\beta_{k} \approx 1$ for $k>3$ ). It is seen from Eqs. (15) and (16) that the "even"-field vortices carrying flux $\phi_{0} / 2$ are by the factor $\lambda_{J} / l \gg 1$ wider than the "odd"-field vortices carrying flux $\phi_{0}$.

Next, we calculate the width of the intervals $\Delta H_{a}$ of existence of the FS states by minimizing free energy, $\mathcal{F}$, of the junction. This can be done explicitly if we specify the spatial distribution of the critical current density, $j_{c}(x)$. Here, for brevity, we use the one harmonic model, i.e., we assume that $j_{c}(x)=j_{1} \sin (2 \pi x / l)$ :

$$
\begin{aligned}
& \mathcal{F}=\left(\frac{H_{a}}{H_{\mathrm{sm}}}\right)^{2}-\frac{\Lambda_{m}}{l} \frac{H_{a}}{H_{\mathrm{sm}}} \frac{4 \pi \phi_{i}}{\phi_{0}}+ \\
& \int_{0}^{L}\left[\Lambda_{m}^{2} \varphi^{\prime 2}-\frac{1}{2} \sin \left(\frac{2 \pi m}{l} x+\theta_{m}\right) \cos \varphi\right] \frac{d x}{L}
\end{aligned}
$$

where $\mathcal{F}$ is normalized by $H_{\mathrm{sm}}^{2} L / 8 \pi$, with $H_{\mathrm{sm}}=$ $\phi_{0} / 4 \pi \lambda \Lambda_{m} \ll H_{m}$. If there is no vortices in the junction, then we have $\psi=0$ and $\varphi(x)=2 \pi \phi_{i} x / \phi_{0} l+\varphi_{0}$, where $\varphi_{0}$ is a constant. In this case the minimum value of the free energy, $\mathcal{F}_{m}$, is achieved for $\varphi_{0}=\theta_{m}$,

$$
\mathcal{F}_{\mathrm{m}}=\left(\frac{2 \pi \Lambda_{m}}{l \phi_{0}}\right)^{2}\left(\phi_{a}-\phi_{i}\right)^{2}-\frac{\sin \left[\pi N\left(m-\phi_{i} / \phi_{0}\right)\right]}{\pi N\left(m-\phi_{i} / \phi_{0}\right)}
$$

where $\phi_{a}=2 \lambda l H_{a}$. Finally, we fix the applied field $H_{a}$ and minimize $\mathcal{F}_{m}$ with respect to the internal flux $\phi_{i}$. It follows from this calculation that if $H_{a}$ is from the narrow interval $m H_{1}-H_{\mathrm{sm}}<H_{a}<m H_{1}+H_{\mathrm{sm}}$, then the flux $\phi_{i}$ is constant and equal to $m \phi_{0}$, which means that $H_{i} \approx m H_{1}$.

The above theoretical analysis can be supported by numerical simulations. To treat both statics and dynamics
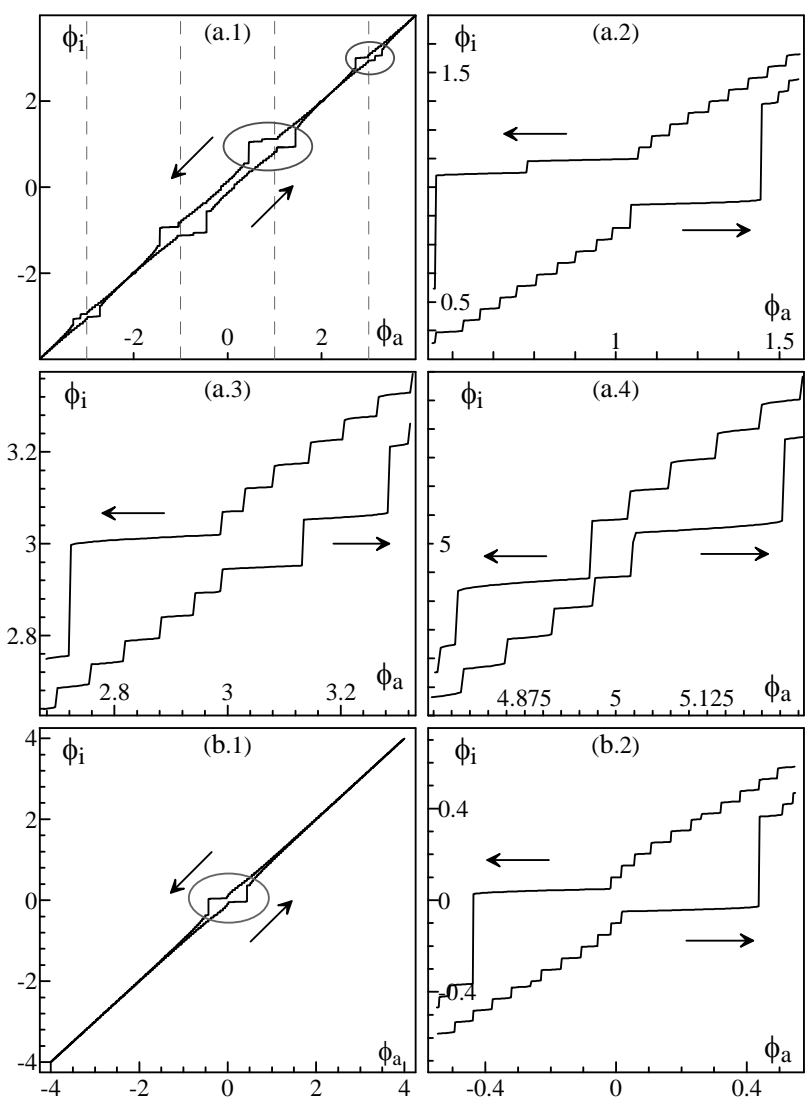

FIG. 3: (a.1) - (a.4) Magnetization, $\phi_{i}\left(\phi_{a}\right)$, of a junction with stepwise critical current density and $N=40 ;$ (a.1) the plateaus with $\phi_{i}=$ const reveal the first four FS states. The curves $\phi_{i}\left(\phi_{a}\right)$ in the vicinity of three FS states: (a.2) $H_{a}=H_{1}$, (a.3) $H_{a}=3 H_{1}$; (a.4) $H_{a}=5 H_{1}$. (b.1)- (b.2) Magnetization of a junction of conventional superconductors. (b.2) $\phi_{i}\left(\phi_{a}\right)$ of a conventional junction in the Meissner state.
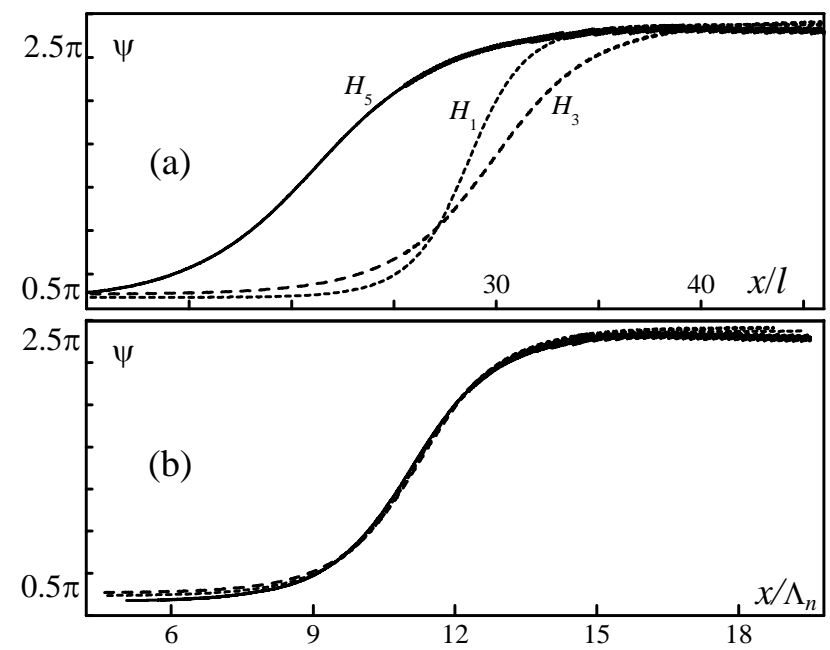

FIG. 4: (a) The phase $\psi(x)$ distribution for vortices carrying flux $\phi_{0}$ at three "odd" resonant fields $H_{1}, 3 H_{1}$, and $5 H_{1}$. (b) The same three curves are shown to collapse into one curve if the coordinate is normalized by $\Lambda_{1}, \Lambda_{3}$, and $\Lambda_{5}$. 


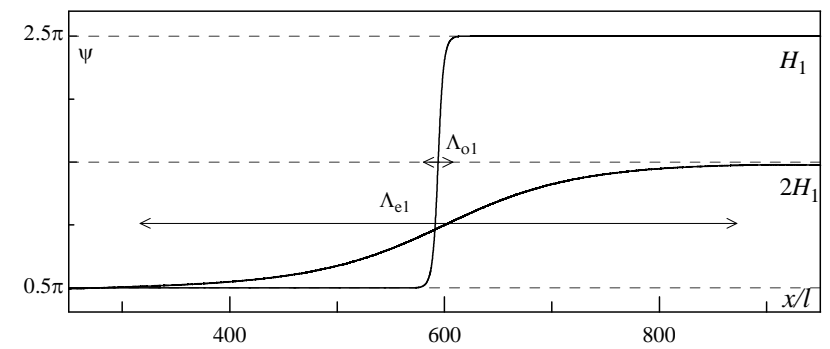

FIG. 5: The phase $\psi(x)$ distribution for the first "odd", $H_{a}=$ $H_{1}$, and "even" $H_{a}=2 H_{1}$ resonant fields describing vortices with fluxes $\phi_{0}\left(H_{a}=H_{1}\right)$ and $\phi_{0} / 2\left(H_{a}=2 H_{1}\right)$.

of the phase difference $\varphi(x, t)$ we introduce time dependence into Eq. (8) and arrive to

$$
\ddot{\varphi}+\alpha \dot{\varphi}-\lambda_{J}^{2} \varphi^{\prime \prime}+i_{c}(x) \sin \varphi=0,
$$

where $\alpha \sim 1$ is a decay constant. The second term in the LHS of Eq. (19) describes dissipation. As a result of this dissipation the system ends up in one of the stable stationary states which is a solution of Eq. (8)).

We used the finite-difference explicit method (see for details [18]) to solve Eq. (19) assuming that the alternating critical current density $j_{c}(x)$ is stepwise. The magnetization curves (dependencies of $\phi_{i}$ on $\phi_{a}$ ) obtained by numerical simulations are shown in Fig. 3. The plateaus with $\phi_{i}=$ const are clearly seen for the series of the four FS states. The field inside the junction is constant for each of the FS states in contrast to junctions of conventional superconductors for which the internal field is constant only in the Meissner state. It is seen in Fig. 3 that the magnetization curve of a junction in the FS state is the same as the magnetization curve of a junction of conventional superconductors but with the field $H_{a}$ biased by $m H_{1}$. Our numerical simulations confirm that the width of the plateau in which the internal field is constant is proportional to $\sqrt{\left|i_{m}\right|}=\lambda_{J} / \Lambda_{m}$ as it follows from the above theoretical analysis.

Next, we simulated numerically the dynamics and statics of single Josephson-type vortices in the FS states (see Figs. 4 and (5). We observed stable Josephson-type vortices carrying fluxes $\phi_{0}$ (for "odd" resonant fields) and $\phi_{0} / 2$ (for "even" resonant fields). The width of vortices carrying flux $\phi_{0}$ scales proportionally to $\sqrt{\left|i_{m}\right|}$ as follows from Eq. (15). Eq. (16) shows that the width of vortices carrying flux $\phi_{0} / 2$ is by factor $\lambda_{J} / l \gg 1$ larger than that of $\phi_{0}$.

We found also that periodical motion of single vortices between the edges of the junction $(x=0$ and $x=L)$ produce steps in the IV characteristics at a series of voltages $V_{m}=m \phi_{0} c_{s} / L$, where $m$ is the number of fluxons inside the junction and $c_{s}$ is the Swihart velocity [16]. These high field steps are similar to the zero field steps in junctions of conventional superconductors [19, 20, 21].

To summarize, we find new equilibrium FS states in
Josephson junctions with periodically alternating critical current density. The FS states exist if the applied field is from narrow intervals centered at equidistant series of resonant fields. If a junction is in FS state, the flux in the junctions is fixed and the maximum supercurrent across the junction is significantly enhanced. Two types of single high-field vortices with flux $\phi_{0}$ or $/$ and $\phi_{0} / 2$ exist in FS states. Periodic motion of these vortices between the edges of the junction results in high field steps in the IV characteristics.

One of the authors (RGM) is grateful to J. R. Clem, V. G. Kogan, J. Mannhart, and C. W. Schneider for support and stimulating discussions.

* mints@post.tau.ac.il

[1] L. N. Bulaevskii, V. V. Kuzii, and A. A. Sobyanin, JETP Letters 25, 290 (1977).

[2] A. I. Buzdin, L. N. Bulaevskii, and S. V. Panyukov, JETP Lett. 35, 178 (1982).

[3] V. V. Ryazanov, V. A. Oboznov, A. Y. Rusanov, A. V. Veretennikov, A. A. Golubov, and J. Aarts, Phys. Rev. Lett. 86, 2427 (2001).

[4] T. Kontos, M. Aprili, J. Lesueur, F. Genet, B. Stephanidis, and R. Boursier, Phys. Rev. Lett. 89, 137007 (2002).

[5] Y. Blum, A. Tsukernik, M. Karpovski, and A. Palevski, Phys. Rev. Lett. 89, 187004 (2002).

[6] C. A. Copetti, F. Rüders, B. Oelze, C. Buchal, B. Kabius, and J. W. Seo, Physica C 253, 63 (1995).

[7] H. Hilgenkamp, J. Mannhart, and B. Mayer, Phys. Rev. B 53, 14586 (1996).

[8] D. J. Van Harlingen, Rev. Mod. Phys. 67, 515 (1995).

[9] C. C. Tsuei and J. R. Kirtley, Rev. Mod. Phys. 74, 969 (2000).

[10] H. Hilgenkamp and J. Mannhart, Rev. Mod. Phys. 74, 485 (2002).

[11] J. Mannhart, H. Hilgenkamp, B. Mayer, C. Gerber, J. R. Kirtley, K. A. Moler, and M. Sigrist, Phys. Rev. Lett. 77, 2782 (1996).

[12] R. G. Mints and V. G. Kogan, Phys. Rev. B 55, R8682 (1997)

[13] R. G. Mints, Phys. Rev. B 57, R3221 (1998).

[14] R. G. Mints, I. Papiashvili, J. R. Kirtley, H. Hilgenkamp, G. Hammerl, and J. Mannhart, Phys. Rev. Lett. 89, 067004 (2002).

[15] A. Buzdin and A. E. Koshelev, Phys. Rev. B 67, 220504(R) (2003).

[16] M. Tinkham, Introduction to Superconductivity (Dover Publications, Inc., Mineola, New York, 2004), 2nd ed.

[17] V. V. Arnold, V. I. Kozlov and A. I. Neishtadt, Mathematical aspects of classical and celestial mechanics (Springer, 1997), 2nd ed.

[18] R. G. Mints and I. Papiashvili, Phys. Rev. B 62, 15214 (2000).

[19] T. A. Fulton and R. C. Dynes, Solid state Communications 12, 57 (1973).

[20] J. T. Chen, T. F. Finnegan, and D. N. Langenberg, Physica 55, 413 (1971).

[21] N. F. Pedersen, Solitons (Elsevier, Amsterdam, 1986). 